\title{
Inflammation Disrupts the LDL Receptor Pathway and Accelerates the Progression of Vascular Calcification in ESRD Patients
}

\author{
Jing Liu ${ }^{1}$, Kun Ling Ma ${ }^{1 *}$, Min Gao ${ }^{1}$, Chang Xian Wang ${ }^{2}$, Jie $\mathrm{Ni}^{1}$, Yang Zhang ${ }^{1}$, Xiao Liang Zhang ${ }^{1}$, \\ Hong Liu' ${ }^{1}$, Yan Li Wang ${ }^{1}$, Bi Cheng Liu' ${ }^{1}$
}

1 Institute of Nephrology, Zhong Da Hospital, Southeast University School of Medicine, Nanjing City, Jiangsu Province, People's Republic of China, 2 Department of Infection Management, Zhong Da Hospital, Southeast University School of Medicine, Nanjing City, Jiangsu Province, People's Republic of China

\begin{abstract}
Background: Chronic inflammation plays a crucial role in the progression of vascular calcification (VC). This study was designed to investigate whether the low-density lipoprotein receptor (LDLr) pathway is involved in the progression of VC in patients with end-stage renal disease (ESRD) during inflammation.

Methods and Results: Twenty-eight ESRD patients were divided into control and inflamed groups according to plasma Creactive protein (CRP) level. Surgically removed tissues from the radial arteries of patients receiving arteriovenostomy were used in the experiments. The expression of tumour necrosis factor- $\alpha$ (TNF- $\alpha$ ) and monocyte chemotactic protein-1 (MCP-1) of the radial artery were increased in the inflamed group. Hematoxylin-eosin and alizarin red $S$ staining revealed parallel increases in foam cell formation and calcium deposit formation in continuous cross-sections of radial arteries in the inflamed group compared to the control, which were closely correlated with increased LDLr, sterol regulatory element binding protein-2 (SREBP-2), bone morphogenetic proteins-2 (BMP-2), and collagen I protein expression, as shown by immunohistochemical and immunofluorescent staining. Confocal microscopy confirmed that inflammation enhanced the translocation of the SREBP cleavage-activating protein (SCAP)/SREBP-2 complex from the endoplasmic reticulum to the Golgi, thereby activating LDLr gene transcription. Inflammation increased alkaline phosphatase protein expression and reduced $\alpha$-smooth muscle actin protein expression, contributing to the conversion of the vascular smooth muscle cells in calcified vessels from the fibroblastic to the osteogenic phenotype; osteogenic cells are the main cellular components involved in VC. Further analysis showed that the inflammation-induced disruption of the LDLr pathway was significantly associated with enhanced BMP-2 and collagen I expression.
\end{abstract}

Conclusions: Inflammation accelerated the progression of VC in ESRD patients by disrupting the LDLr pathway, which may represent a novel mechanism involved in the progression of both VC and atherosclerosis.

Citation: Liu J, Ma KL, Gao M, Wang CX, Ni J, et al. (2012) Inflammation Disrupts the LDL Receptor Pathway and Accelerates the Progression of Vascular Calcification in ESRD Patients. PLoS ONE 7(10): e47217. doi:10.1371/journal.pone.0047217

Editor: Alberico Catapano, University of Milan, Italy

Received June 1, 2012; Accepted September 10, 2012; Published October 24, 2012

Copyright: (C) 2012 Liu et al. This is an open-access article distributed under the terms of the Creative Commons Attribution License, which permits unrestricted use, distribution, and reproduction in any medium, provided the original author and source are credited.

Funding: This work was supported by the Natural Science Foundation of Jiangsu Province (BK2009279) and the National Natural Science Foundation of China (Grants 81170792 and 81070571 ). The funding agencies had no role in the study design, data collection and analysis, decision to publish, or preparation of the manuscript.

Competing Interests: The authors have declared that no competing interests exist.

* E-mail: mmkkll@hotmail.com

\section{Introduction}

Cardiovascular disease (CVD) is the leading cause of morbidity among patients with end-stage renal disease (ESRD), accounting for approximately $50 \%$ of deaths and $30 \%$ of hospitalisations in this population [1]. Annual CVD mortality is 10-20 fold higher in ESRD patients than in the general population, and this difference is not completely explained by traditional risk factors [2]. Recently, more attention has been paid to vascular calcification (VC), which induces arterial stiffness, high pulse pressure, and cardiac valve dysfunction, contributing to ventricular hypertrophy and heart failure [3,4]. Thus, VC results in an increased risk of CVD mortality, especially in ESRD patients, regardless of maintenance hemodialysis (HD) treatment status.
Vascular calcification is a complicated pathological process that develops primarily within the intimal and medial layers of the artery. Arterial intimal calcification (AIC) is an advanced form of atherosclerosis (AS), driven by cellular necrosis, inflammation, and lipid deposition manifested in a patchy, discontinuous course along the artery. Specific risk factors for AIC in uraemia patients include hyperphosphatemia, hypoalbuminemia, excessive calcium intake, and HD duration. Arterial medial calcification (AMC) is observed in the elastic lamella of the medial layer of the arteries. AMC is closely associated with HD duration even in patients with no CVD history at HD therapy onset. AMC is an active process that involves the transformation of medial vascular smooth muscle cells (VSMCs) from a fibroblastic to an osteogenic phenotype. Normally, VSMCs have a contractile phenotype and constitutively 
express proteins that inhibit mineralisation. In response to various stimuli, however, VSMCs express and/or release several key regulators of bone formation and bone structural associated proteins, such as bone morphogenetic protein-2 (BMP-2), alkaline phosphatase (ALP), and collagen I. In contrast, the expression of proteins such as $\alpha$-smooth muscle cell ( $\alpha$-SMA) and collagen IV is reduced, ultimately transforming VSMCs into osteoblast-like cells $[5,6]$. However, the precise mechanisms that cause the osteogenic phenotype of VSMCs in calcified vessels are not completely clear.

Chronic systemic inflammation is a common feature in ESRD patients [7], and it may be correlated with the accumulation of pro-inflammatory compounds caused by a markedly decreased glomerular filtration rate (GFR) [8]. Other causes, including malnutrition, metabolic acidosis, hyperparathyroidism, the accumulation of advanced oxidation protein products and asymmetric dimethyl arginine, contribute to the release of inflammatory cytokines [9]. Inflammation accelerates the progression of AS and VC $[10,11]$, which has been identified as an independent risk factor for the morbidity and mortality of CVD in ESRD patients [12].

It is well known that the low-density lipoprotein receptor (LDLr) pathway is a feedback system with important roles in regulating plasma and intracellular cholesterol homeostasis, and it is mainly modulated by the concentration of intracellular cholesterol and the interaction between sterol regulatory element binding protein (SREBP) and SREBP cleavage-activating protein (SCAP). Cholesterol deficiency enhances the translocation of SCAP from the endoplasmic reticulum (ER) to the Golgi, where it cleaves SREBP, thus increasing LDLr gene expression.

Our previous studies demonstrated that inflammation accelerated the progression of AS by disrupting LDLr feedback regulation $[13,14]$. The present study was performed to evaluate whether the inflammation exacerbates the progression of $\mathrm{VC}$ in ESRD patients and explore the underlying mechanisms.

\section{Materials and Methods}

\section{Ethics Statement}

All studies were approved by the Ethical Committee of Southeast University. Each patient provided written informed consent to the use of their tissues for research purposes.

\section{Patient Selection and Clinical Data}

We studied 28 ESRD patients from Zhong Da Hospital, Southeast University between January 2010 and May 2011. Patients with ESRD who were to undergo arteriovenostomy before hemodialysis were included in the study. Patients with acute infection, cancer, and/or chronic active hepatitis were excluded. The included patients were divided into two groups based on plasma C-reactive protein (CRP) levels: control (CRP $<3.0 \mathrm{mg} / \mathrm{l}$, $\mathrm{n}=14)$ and inflamed $(\mathrm{CRP}>=3.0 \mathrm{mg} / \mathrm{l}, \mathrm{n}=14)$ group. Inflamed group was defined as the patients with persistent increased plasma levels of CRP checked at the start and the second week after hospitalization. The patients were comprehensively monitored by the symptoms, signs, and serum indexes, in order to detect timely any confounding condition which may potentially affect serum CRP level.

\section{Clinical Biochemical Tests}

Blood samples were assayed to determine erythrocyte sedimentation rate (ESR), CRP, red blood cells (RBC), haemoglobin ( $\mathrm{Hb}$ ), total protein (TP), albumin (ALB), glucose (GLU), triglyceride (TG), total cholesterol (TC), low density lipoprotein (LDL), high density lipoprotein (HDL), apolipoprotein Al (Apo A1), Apo B, lipoprotein $(\mathrm{a})$, calcium $(\mathrm{Ca})$, phosphate $(\mathrm{P})$, and intact parathyroid hormone (iPTH) using an automatic biochemistry analyser at the clinical chemistry centre of the hospital.

\section{Tissue Processing}

Tissues from the radial artery were taken during radial-cephalic anastomosis surgery. The tissue sections were rinsed with saline and placed in $10 \%$ buffered formalin. After treatment, representative sections of the grafts were obtained and embedded in O.C.T. medium or paraffin.

\section{Hematoxylin-eosin (HE) Staining}

The paraffin embedded tissues were sliced and dewaxed. The slices were dyed for 15 minutes with hematoxylin, dipped in $1 \%$ hydrochloric alcohol, and then stained with $1 \%$ eosin for 3 minutes. The slices were sealed with resinene after they were dehydrated to transparency. The results were observed under light microscope $(\times 200)$.

\section{Alizarin red S Staining}

The paraffin-embedded tissues were dewaxed and hydrated in $70 \%$ alcohol. After rinsing rapidly in distilled water, the slices were dyed with alizarin red $\mathrm{S}$ solution for one minute. Positive results were shown in an orange-red colour in light microscope $(\times 200)$.

\section{Immunohistochemical Staining}

Paraffin-embedded sections $(4 \mu \mathrm{m})$ were subjected to immunohistochemical staining. After deparaffinisation, sections were placed in citrate-buffered solution $(\mathrm{pH}$ 6.0) and then heated for antigen retrieval. Endogenous peroxidase was blocked with $3 \%$ hydrogen peroxide, and nonspecific antibody binding was blocked with $10 \%$ goat serum. Subsequently, sections were incubated with goat, rabbit or mouse anti-human primary antibodies against TNF- $\alpha$ (Santa Cruz, USA), MCP-1 (Santa Cruz, USA), LDLr (Abcam, UK), BMP-2 (Santa Cruz, USA), and collagen I (Abcam, UK) overnight at $4^{\circ} \mathrm{C}$, followed by incubation with biotinylated secondary antibodies. Finally, a diaminobenzidine tetrahydrochloride substrate was used to develop the reaction. The results were observed under a light microscope $(\times 200)$. Semiquantitative analysis was performed by the software of Image-Pro Plus version 5.0.

\section{Immunofluorescent Staining}

The frozen sections were fixed with $4 \%$ formalin solution and then blocked with $5 \%$ bovine serum albumin (BSA). Subsequently, the sections were incubated with rabbit, mouse, or goat antihuman primary antibodies against SCAP (Abcam, UK), $\alpha$-SMA (Abcam, UK), Golgi (Invitrogen, USA), and ALP (Santa Cruz, USA), followed by goat anti-rabbit Fluor 488, donkey anti-rabbit Fluor 594, goat anti-mouse Fluor 594, and donkey anti-goat Fluor 488 secondary uorescent antibodies (Invitrogen, USA), respectively. After washing, the slides were examined by laser confocal microscopy $(\times 100)$. Semi-quantitative analysis was performed by the software of Image-Pro Plus version 5.0.

\section{Statistical Analysis}

All the data were expressed as the mean \pm standard deviation (SD) and were analysed with SPSS 13.0. Continuous variables were compared between the two groups with the independentsample $t$ test (where appropriate), and correlations between variables were analysed by Spearman's R coefficient. A difference was considered significant if the $P$ value was less than 0.05 . 


\section{Results}

Basic Clinical Data of the Patients in the Two Groups

As shown in Table 1, there were no differences in age, body weight, ESR, RBC, Hb, TP, ALB, lipid profiles, Ca, P, Ca $\times$ P, or iPTH $(\mathrm{P}>0.05)$ between the inflamed group and the control group.

\section{Local Upregulation of Inflammation in the Artery was Consistent with Plasma CRP Level}

Using immunohistochemical staining, we demonstrated that the expression of TNF- $\alpha$ and MCP-1 were increased in the radial artery in the inflamed group, which indicated that the local inflammation in the artery was upregulated, consistent with the observation of systemic inflammation stress (Fig. 1A, Fig. 1B).

\section{Inflammation Induced Foam Cell Formation and Calcified Plaque Deposition of Radial Arteries}

HE staining showed that there was significant foam cell formation in the radial arteries of the inflamed group compared to the control (Fig. 2A, Fig. 2B). Interestingly, there was a parallel increase in calcified plaque deposition in the radial arteries of the inflamed group compared to controls, as evaluated by alizarin red $\mathrm{S}$ staining, suggesting that a common mechanism could be involved in both AS and VC (Fig. 2C, Fig. 2D).

\section{Inflammation Disrupted the Feedback Regulation of the LDL Receptor}

To explore the potential mechanisms underlying this phenomenon, we evaluated the effects of inflammation on the protein expression of LDLr and SREBP-2 by immunohistochemical staining in radial arteries. Inflammation significantly increased LDLr and SREBP-2 protein expression (Fig. 3A I-VI, Fig. 3B).

Table 1. Basic clinical and biochemical data for the patients.

\begin{tabular}{lll}
\hline & & \\
\hline Parameters & control $(\mathbf{n}=\mathbf{1 4})$ & inflamed group $(\mathbf{n}=\mathbf{1 4})$ \\
\hline Weight $(\mathrm{Kg})$ & $63.71 \pm 9.82$ & $63.54 \pm 12.47$ \\
Age $(\mathrm{ys})$ & $49.07 \pm 16.34$ & $57.71 \pm 17.32$ \\
$\mathrm{RBC}\left(10^{12} / \mathrm{L}\right)$ & $2.57 \pm 0.57$ & $2.72 \pm 0.63$ \\
$\mathrm{Hb}(\mathrm{g} / \mathrm{L})$ & $76.89 \pm 17.39$ & $78.67 \pm 18.29$ \\
$\mathrm{TP}(\mathrm{g} / \mathrm{L})$ & $57.07 \pm 10.51$ & $57.36 \pm 9.6$ \\
$\mathrm{ALB}(\mathrm{g} / \mathrm{L})$ & $28.14 \pm 6.20$ & $28.36 \pm 4.89$ \\
$\mathrm{GLU}(\mathrm{mmol} / \mathrm{L})$ & $5.34 \pm 2.46$ & $5.52 \pm 1.92$ \\
$\mathrm{TG}(\mathrm{mmol} / \mathrm{L})$ & $1.33 \pm 1.06$ & $1.18 \pm 0.64$ \\
$\mathrm{~T}-\mathrm{CHO}(\mathrm{mmol} / \mathrm{L})$ & $4.81 \pm 1.26$ & $4.51 \pm 1.00$ \\
$\mathrm{LDL}(\mathrm{mmol} / \mathrm{L})$ & $3.09 \pm 1.09$ & $2.83 \pm 0.87$ \\
$\mathrm{HDL}(\mathrm{mmol} / \mathrm{L})$ & $1.21 \pm 0.28$ & $1.08 \pm 0.37$ \\
$\mathrm{ApoA} 1(\mathrm{mmol} / \mathrm{L})$ & $1.11 \pm 0.23$ & $0.97 \pm 0.29$ \\
$\mathrm{ApoB}(\mathrm{mmol} / \mathrm{L})$ & $0.89 \pm 0.30$ & $0.81 \pm 0.21$ \\
$\mathrm{LP}(\mathrm{a})(\mathrm{mmol} / \mathrm{L})$ & $395.43 \pm 204.79$ & $366.43 \pm 203.45$ \\
$\mathrm{Ca}(\mathrm{mmol} / \mathrm{L})$ & $2.00 \pm 0.24$ & $2.01 \pm 0.27$ \\
$\mathrm{P}(\mathrm{mmol} / \mathrm{L})$ & $1.89 \pm 0.31$ & $1.83 \pm 0.47$ \\
$\mathrm{Ca}$ *P $(\mathrm{mmol} / \mathrm{L}){ }^{2}$ & $3.75 \pm 0.55$ & $3.57 \pm 0.54$ \\
$\mathrm{iPTH}(\mathrm{pg} / \mathrm{mL})$ & $255.84 \pm 168.57$ & $205.05 \pm 133.97$ \\
\hline & & \\
\hline
\end{tabular}

There was no difference compared every index in the inflamed group with that in the control, $P>0.05$.

doi:10.1371/journal.pone.0047217.t001
Moreover, the plasma CRP level was positively correlated with the expression of the LDLr protein (Fig. 3G). Therefore, we further investigated the effect of inflammation on the translocation of SCAP escorting SREBP-2 from the ER to the Golgi in the radial arteries. Confocal microscopy showed that inflammation significantly increased SCAP translocation from the ER to the Golgi, thereby activating LDLr gene transcription (Fig. 3D, Fig. 3E.).

\section{Inflammation Accelerated VC by Contributing to VSMC Conversion from the Fibroblastic to the Osteogenic Phenotype}

To investigate the possible mechanisms of $\mathrm{VC}$ in the context of inflammation, we evaluated the effects of inflammation on the expression of the bone formation biomarkers BMP-2 and collagen $\mathrm{I}$ in the radial arteries during $\mathrm{VC}$ progression. As shown by immunohistochemical staining, BMP-2 and collagen I protein expression were significantly increased in the inflamed group compared to the control group (Fig. 4A I-VI, Fig. 4B). It is well known that VSMC is one of the major cellular components involved in the progression of AMC. Therefore, we evaluated the protein expressions of ALP and $\alpha$-SMA. As shown by immunofluorescent staining, inflammation significantly increased ALP expression and decreased $\alpha$-SMA expression (Fig. 4C, Fig. 4D). This suggests that inflammation induces VSMC conversion from the fibroblastic to the osteogenic phenotype, thereby accelerating the progression of AMC.

\section{The Disruption of the LDLr Pathway was Closely Associated with AS and VC of the Radial Arteries}

Correlation analysis demonstrated that LDLr protein expression was positively associated with BMP-2 and collagen I protein expression (Fig. 5A and Fig. 5B). These findings, in combination with those of our previous studies, suggest that the disruption of the LDLr pathway under inflammatory stress may be closely associated with the progression of $\mathrm{AS}$ and VC.

\section{Discussion}

Dyslipidemia and chronic inflammation are common complications in chronic kidney disease (CKD), especially ESRD. It has been reported that dyslipidemia and inflammation act together as "partners in crime" to accelerate the progression of AS and vascular calcification in HD patients. Our previous in vivo and in vitro studies showed that inflammation induced intracellular lipid accumulation and foam cell formation by disrupting LDLr feedback regulation, exacerbating the progression of AS [13-15]. The present study was designed to investigate whether the LDLr pathway was involved in the progression of $\mathrm{VC}$ in $\mathrm{HD}$ patients under inflammatory stress.

Using immunohistochemical staining, we found that TNF- $\alpha$ and MCP-1 protein expression were increased in the arteries of the inflamed group compared to controls, suggesting that local arterial inflammation was also upregulated in the inflamed group.

By evaluating continuous cross-sections, we demonstrated that there was a significant increase in foam cell formation and calcium deposition in the inflamed group, as shown by hematoxylin eosin and alizarin red S staining, respectively. The parallel pathological changes in $\mathrm{AS}$ and $\mathrm{VC}$ appeared in the same area of the radial artery, suggesting that a common pathway mediated by inflammation and dyslipidemia may be involved in the progression of both AS and VC.

We found that LDLr protein expression in the radial artery tissues was increased in the inflamed group compared to the control group and that this increase was positively associated with 
A

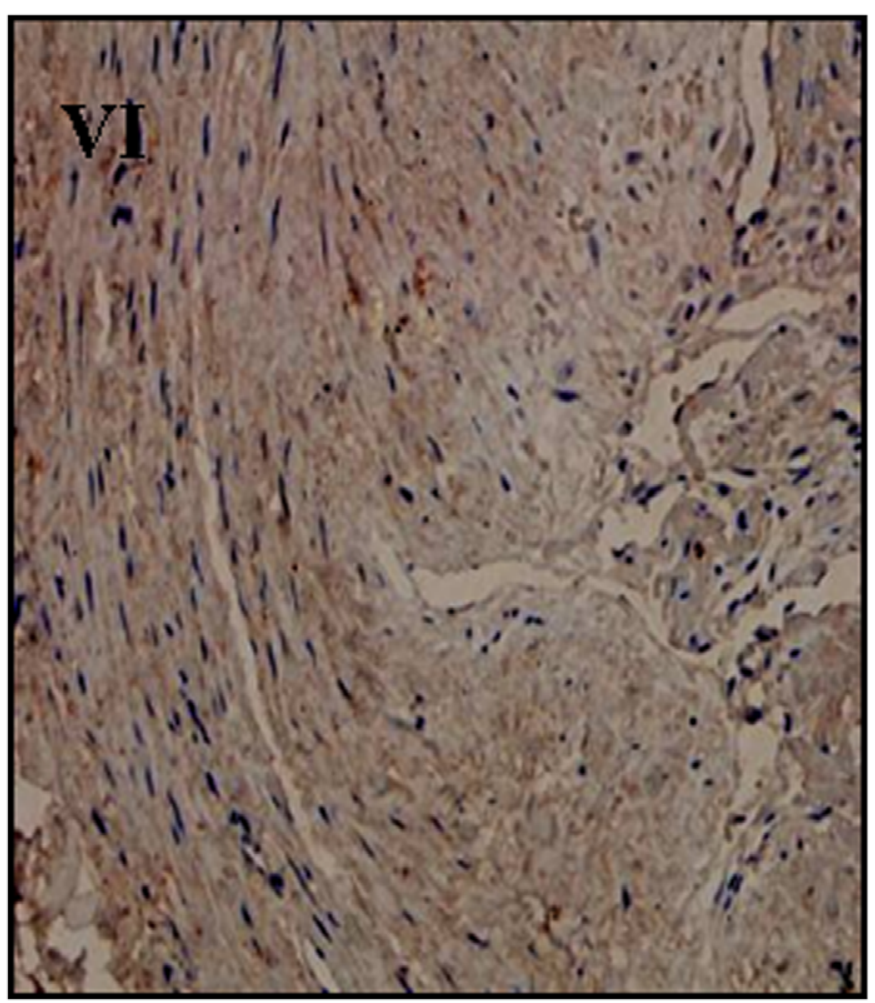

B

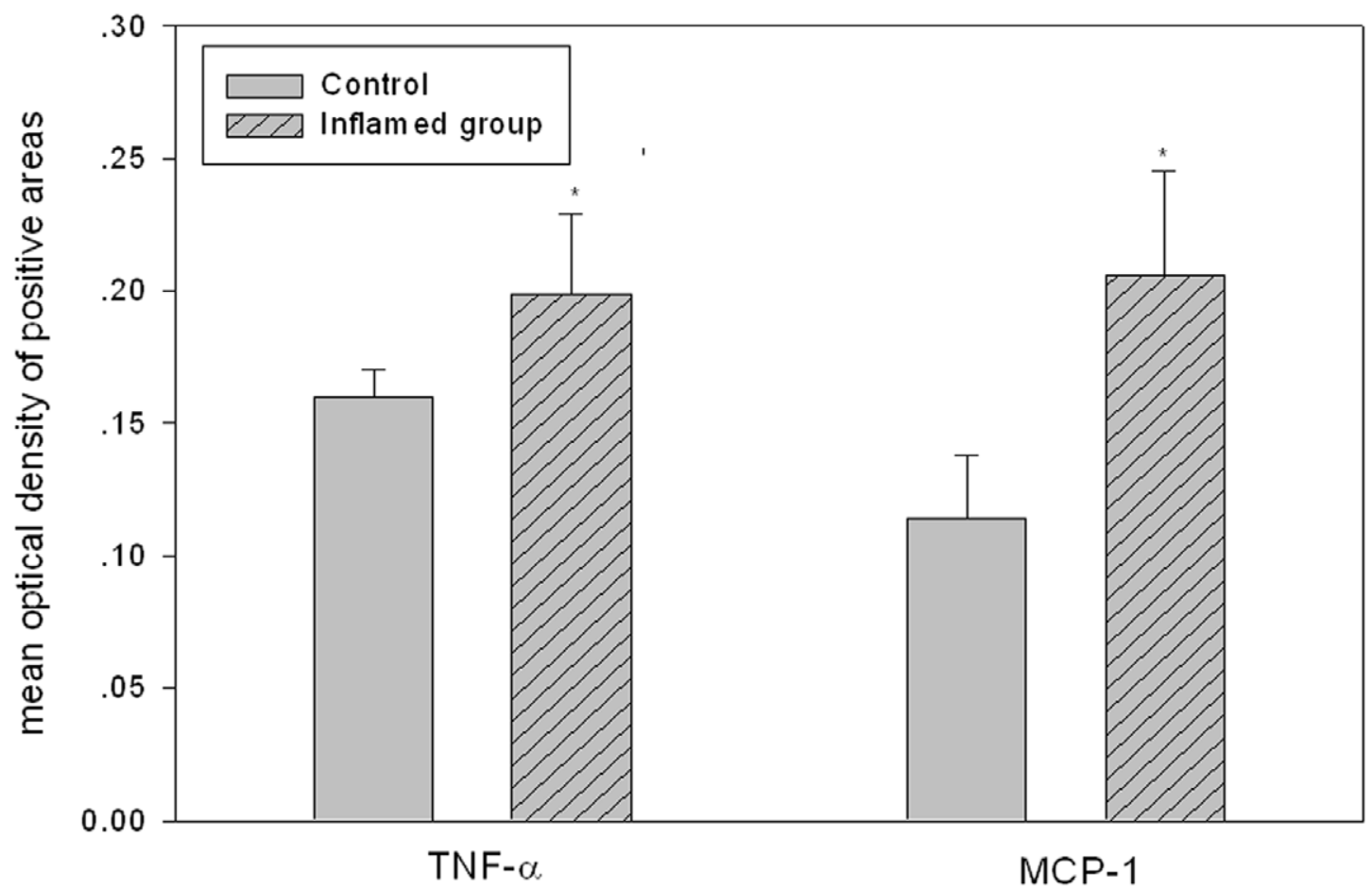

Figure 1. Locally upregulated inflammation in the artery was consistent with the plasma CRP level. The local inflammation status in the radial artery was examined by immunohistochemical staining. The positive areas were stained brown in cross-sections of radial arteries (Fig. 1, I-VI, original magnification $\times 200$ ). The values of semiquantitative analysis for the positive areas were expressed as the mean \pm SD from five patients in each group $(\mathrm{n}=14) .{ }^{*} P<0.05$ vs control (Fig. 1B).

doi:10.1371/journal.pone.0047217.g001 


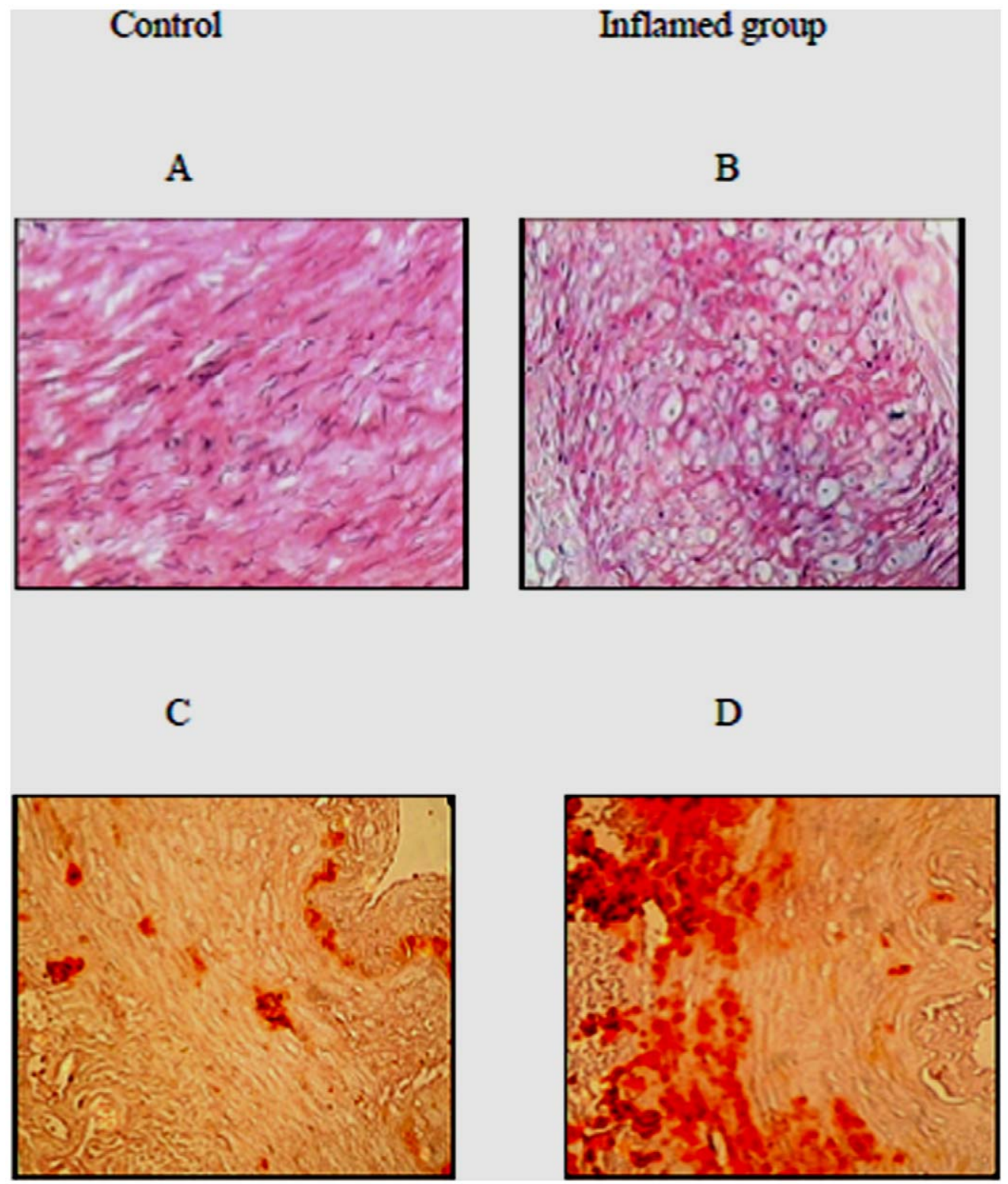

Figure 2. Inflammation induced foam cell formation and calcified plaque deposition in the radial arteries. The lipid accumulation in the radial arteries was checked by hematoxylin-eosin staining (Fig. $2 \mathrm{~A}$ and Fig. $2 \mathrm{~B}$, original magnification $\times 200$ ) Calcification was examined by alizarin red $\mathrm{S}$ staining, and calcium deposits were stained orange-red (Fig. 2C and Fig. 2D, original magnification $\times 200$ ). doi:10.1371/journal.pone.0047217.g002

plasma CRP level. Further analysis showed that upregulated LDLr protein expression was mediated by increased SREBP-2 protein expression and enhanced SCAP/SREBP-2 complex translocation. These clinical findings were consistent with our previous in vivo and in vitro studies, showing that the disruption of the LDLr pathway played crucial roles in the progression of AS.

It is accepted that AMC, the main calcification mechanism in CKD patients, is closely associated with the conversion of VSMCs from fibroblastic to osteogenic phenotypes and the upregulation of osteogenic programs [16]. Although Proudfoot et al reported that acetylated LDL stimulates human VSMC calcification by promoting osteoblastic differentiation, little is known about the roles of LDLr feedback regulation in modulating the phenotype conversion of VSMCs in calcified vessels in the context of inflammation. To investigate the possible link between the disruption of the LDLr pathway and VC, we further evaluated the effects of inflammation on AMC in the tissues of the radial artery. Using tissues removed from the radial arteries of HD patients, we demonstrated that inflammation significantly increased the expression of the bone formation biomarker proteins BMP-2 and collagen I during VC progression. Immunofluorescent staining showed that inflammation significantly increased ALP expression and decreased $\alpha$-SMA expression. The correlation analysis further showed that LDLr protein expression was positively associated with BMP-2 and collagen I protein expression. These observations suggest that inflammation accelerates the progression of AMC by disrupting the LDLr pathway, which is closely associated with the induction of VSMC phenotype conversion. Recently, using LDL receptor deficient mice, Geng $\mathrm{Y}$ et al [17] demonstrated that up-regulation of cholesterol 
A

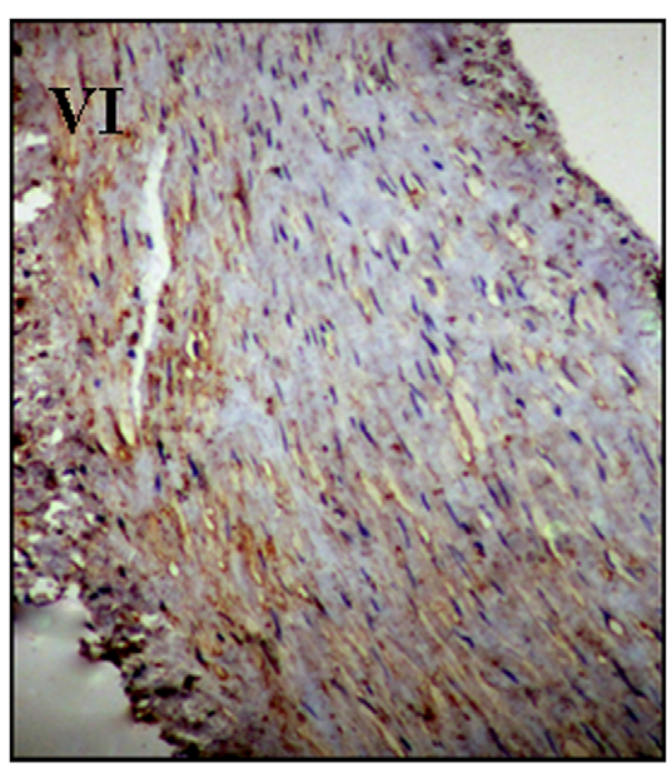

C

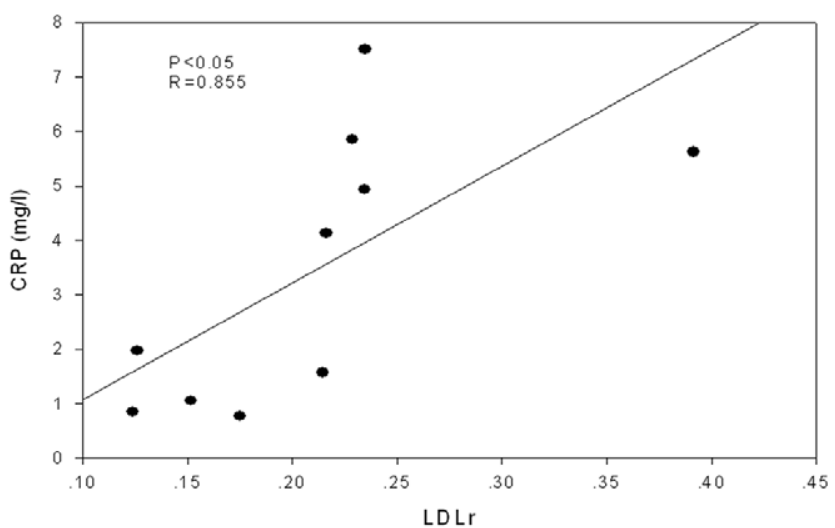

$\mathbf{E}$

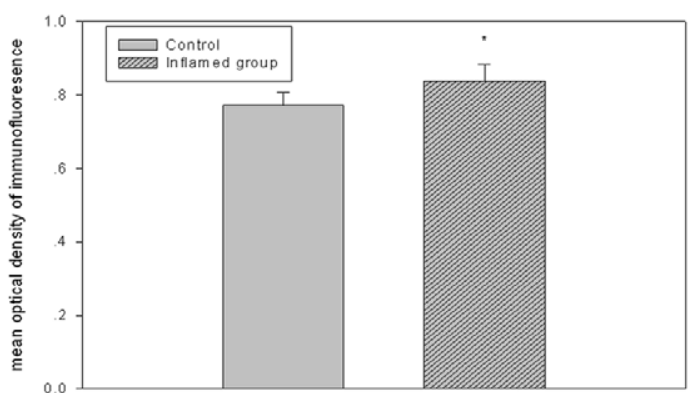

B

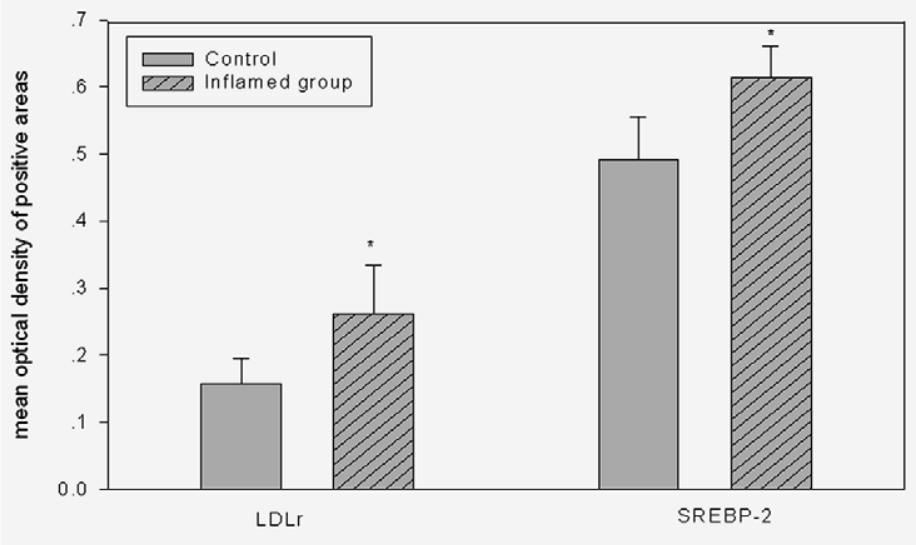

D

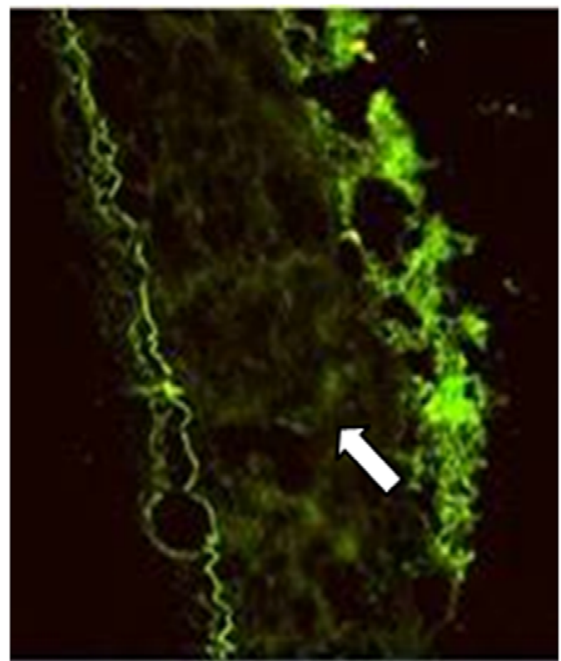

Figure 3. Inflammation disrupted the feedback regulation of the LDL receptor. LDLr and SREBP-2 protein expression were measured by immunohistochemical staining. The positive areas were stained brown in radial artery cross sections (Fig. 3A I-VI, original magnification $\times 200$ ). The 
values of semiquantitative analysis for the positive areas are expressed as the mean \pm SD from five patients at each group ( $n=14)$. ${ }^{*} P<0.05$ vs control (Fig. 3B). Correlation analysis of plasma CRP level with LDLr expression (Fig. 3C). The translocation of SCAP from the ER to Golgi was evaluated by immunofluorescent staining. SCAP and Golgi are stained in green and red, respectively. The colocalisation of SCAP with Golgi was evaluated by laser confocal microscopy (Fig. 3D, arrow indicates colocalisation, original magnification $\times 100$ ). Overlay areas were quantified and expressed as the mean \pm SD from five patients at each group $(n=14) .{ }^{*} P<0.05$ vs control (Fig. 3E)

doi:10.1371/journal.pone.0047217.g003

A

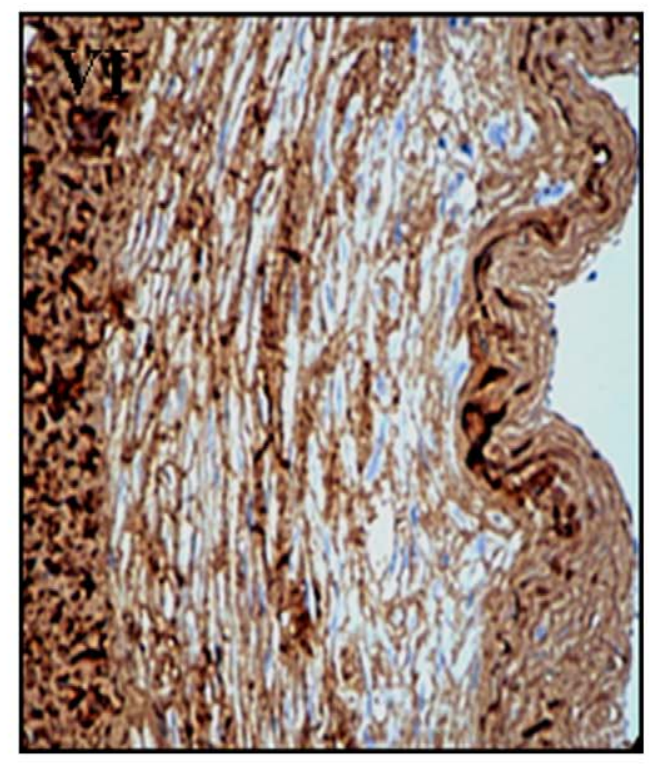

C

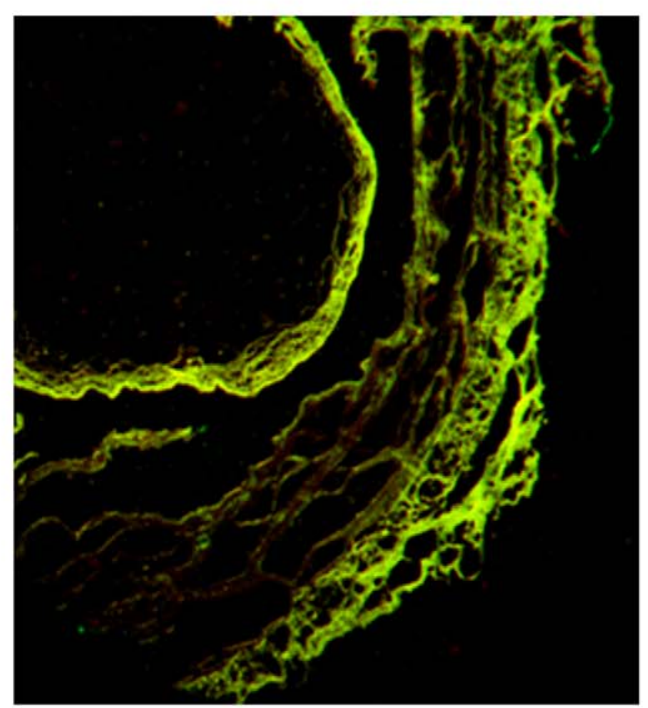

B

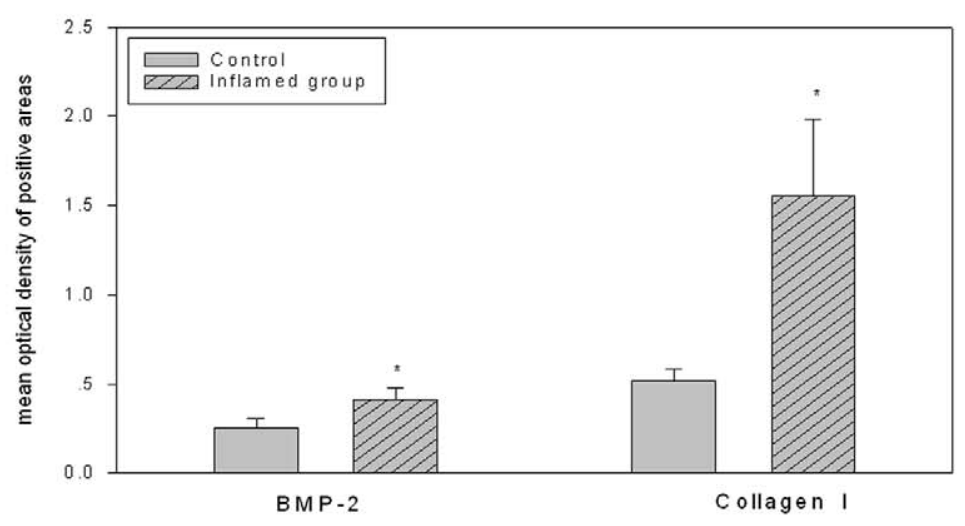

D

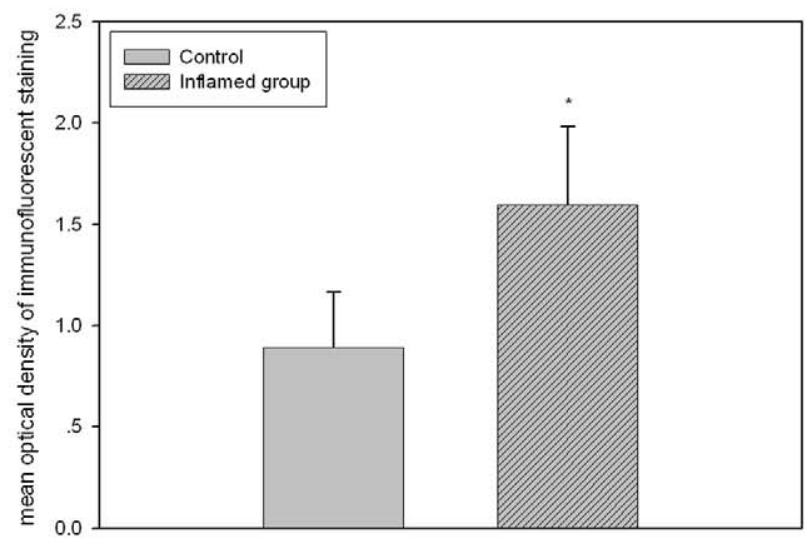

Figure 4. Inflammation accelerated the VC by contributing to the phenotype conversion of VSMC from the fibroblastic to the osteogenic. The protein expressions of BMP-2 and Collagen I were checked by immunohistochemical staining. The positive areas were stained as brown in cross-sections of radial arteries (Fig. 4A I-VI, original magnification $\times 200$ ). The values of semiquantitative analysis for the positive areas were expressed as the mean \pm SD from five patients at each group $(n=14)$. ${ }^{*} P<0.05$ vs control (Fig. 4B). The co-expression of the ALP and $\alpha$-SMA proteins was checked by immunofluorescent double staining. ALP and $\alpha$-SMA are stained with green and red fluorescence, respectively (Fig. 4C, original magnification $\times 100$ ). The ratio of green to red in the overlay areas were quantified and expressed as the mean \pm SD from five patients in each group $(n=14) . * P<0.05$ vs control (Fig. 4D).

doi:10.1371/journal.pone.0047217.g004 
A

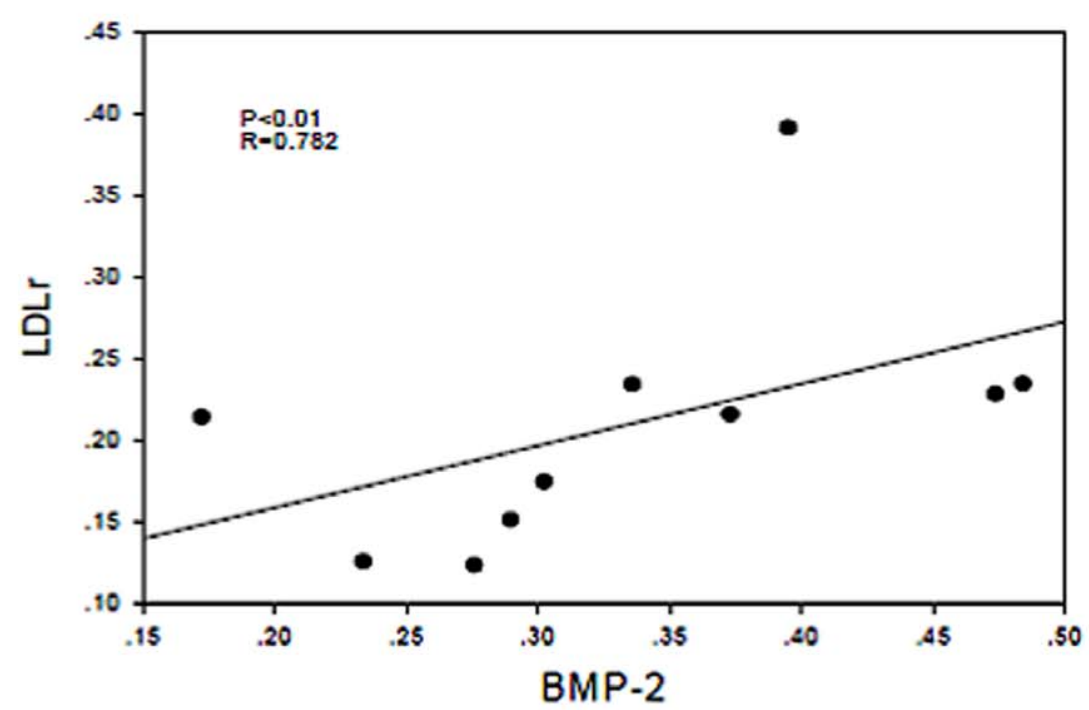

B

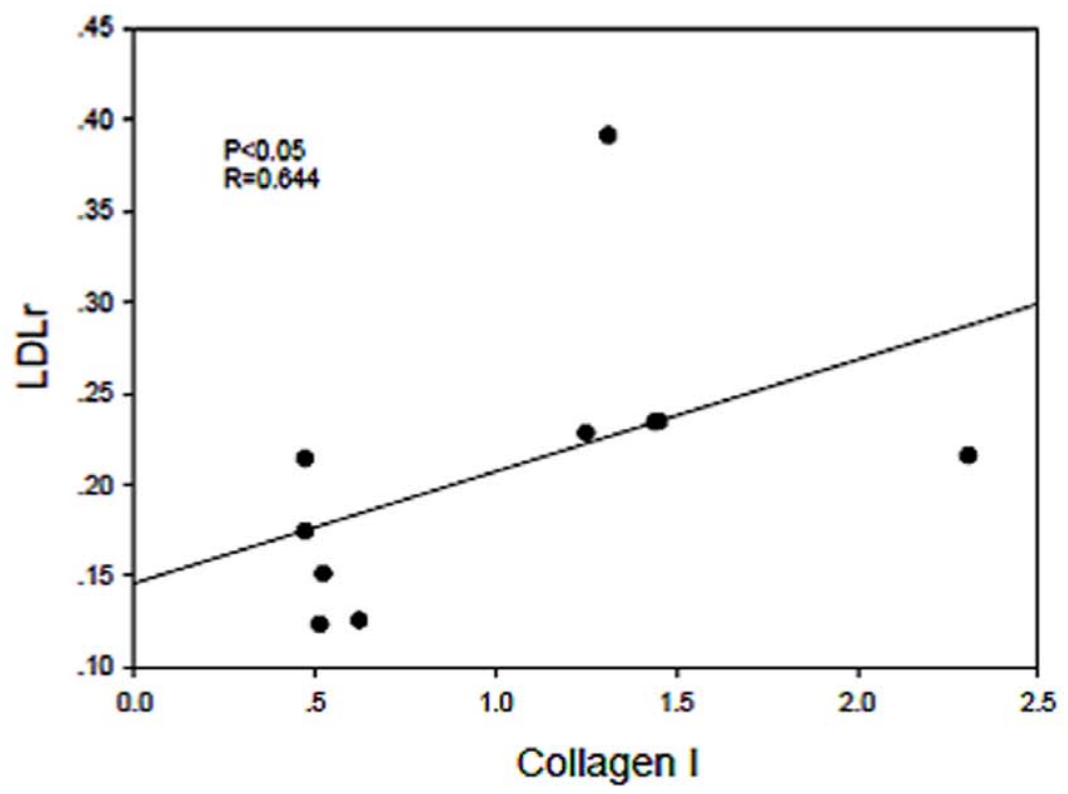

Figure 5. The disruption of the LDLr pathway was closely associated with VC of the radial arteries. Correlation analysis demonstrated that LDLr protein expression was positively associated with BMP-2 (Fig. $5 A, R=0.782, P<0.01$ ) and collagen I (Fig. $5 B, R=0.644, P<0.05$ ) expression. doi:10.1371/journal.pone.0047217.g005

metabolism is essential for matrix mineralization by vascular cells, which were further confirmed by our findings.

In summary, our findings demonstrate for the first time that inflammation accelerates the progression of $\mathrm{VC}$ in $\mathrm{HD}$ patients by disrupting the LDLr pathway. This may be a novel mechanism involved in the progression of both $\mathrm{VG}$ and $\mathrm{AS}$.

\section{Limitations}

The main limitation was low number of patients and small size of samples acquired from radial arteries in two groups, which may limit providing more evidence with the evaluation of VC. CRP, as a biomarker in this study for the evaluation of inflammatory status in ESRD patients, should be understood objectively. Although it has been widely accepted as a valuable and well recognized index of inflammation, some studies recently have reported that CRP partly depends upon commonly unmeasured factors (e. g. genetic traits) [18,19]. In addition, as each patient had different conditions, such as age, complications, treatments, serum levels of phosphate, calcium, and intact parathyroid hormone, the data could be not well-controlled.

\section{Author Contributions}

Conceived and designed the experiments: KLM. Performed the experiments: JN J. Liu Y. Zhang. Analyzed the data: KLM C.X. Wang B.C. Liu. Contributed reagents/materials/analysis tools: KLM J. Liu. Wrote the paper: MG H. Liu X.L. Zhang Y.L. Wang. 


\section{References}

1. Locatelli F, Pisoni RL, Combe C, Bommer J, Andreucci VE, et al (2004) Anaemia in haemodialysis patients of five European countries: association with morbidity and mortality in the Dialysis Outcomes and Practice Patterns Study (DOPPS). Nephrol Dial Transplant 19: 121-132.

2. Foley RN, Parfrey PS, Sarnak MJ (1998) Clinical epidemiology of cardiovascular disease in chronic renal disease. Am J Kidney Dis 32: S112-S119.

3. London GM, Guerin AP, Marchais SJ, Metivier F, Pannier B, et al (2003) Arterial media calcification in end-stage renal disease: impact on all-cause and cardiovascular mortality. Nephrol Dial Transplant 18: 1731-1740.

4. London GM (2003) Cardiovascular calcifications in uremic patients: clinical impact on cardiovascular function. J Am Soc Nephrol 14: S305-S309.

5. Giachelli GM (2004) Vascular calcification mechanisms. J Am Soc Nephrol 15: 2959-2964.

6. Shanahan CM, Crouthamel MH, Kapustin A, Giachelli CM (2011) Arterial calcification in chronic kidney disease: key roles for calcium and phosphate. Circ Res 109: 697-711.

7. Kaysen GA (2001) The microinflammatory state in uremia: causes and potential consequences. J Am Soc Nephrol 12: 1549-1557.

8. Akahoshi T, Kobayashi N, Hosaka S, Sekiyama N, Wada C, et al (1995) In-vivo induction of monocyte chemotactic and activating factor in patients with chronic renal failure. Nephrol Dial Transplant 10: 2244-2249.

9. Silverstein DM (2009) Inflammation in chronic kidney disease: role in the progression of renal and cardiovascular disease. Pediatr Nephrol 24: 1445-1452.

10. Hansson GK, Robertson AK, Soderberg-Naucler C (2006) Inflammation and atherosclerosis. Annu Rev Pathol 1: 297-329.

11. Moe SM, Chen NX (2005) Inflammation and vascular calcification. Blood Purif 23: $64-71$.
12. Zimmermann J, Herrlinger S, Pruy A, Metzger T, Wanner C (1999) Inflammation enhances cardiovascular risk and mortality in hemodialysis patients. Kidney Int 55: 648-658.

13. Ma KL, Ruan XZ, Powis SH, Moorhead JF, Varghese Z (2007) Antiatherosclerotic effects of sirolimus on human vascular smooth muscle cells. Am J Physiol Heart Circ Physiol 292: H2721-H2728.

14. Ruan XZ, Moorhead JF, Tao JL, Ma KL, Wheeler DC, et al (2006) Mechanisms of Dysregulation of Low-Density Lipoprotein Receptor Expression in Vascular Smooth Muscle Cells by Inflammatory Cytokines. Arterioscler Thromb Vasc Biol 26(5): 1150-5.

15. Ma KL, Ruan XZ, Powis SH, Chen Y, Moorhead JF, et al (2008) Inflammatory stress exacerbates lipid accumulation in hepatic cells and fatty livers of apolipoprotein E knockout mice. Hepatology 48: 770-781.

16. Amann K (2008) Media calcification and intima calcification are distinct entities in chronic kidney disease. Clin J Am Soc Nephrol 3: 1599-1605.

17. Geng Y, Hsu JJ, Lu J, Ting TC, Miyazaki M, et al (2011) Role of cellular cholesterol metabolism in vascular cell calcification. J Biol Chem 286: 3370133706.

18. Crawford DC, Sanders CL, Oin X, Smith JD, Shephard C, et al (2006) Genetic variation is associated with C-reactive protein levels in the Third National Health and Nutrition Examination Survey. Circulation 114: 2458-2465.

19. Thalmaier D, Dambacher J, Seiderer J, Konrad A, Schachinger V, et al (2006) The $+1059 \mathrm{G} / \mathrm{C}$ polymorphism in the C-reactive protein (CRP) gene is associated with involvement of the terminal ileum and decreased serum CRP levels in patients with Crohn's disease. Aliment Pharmacol Ther 24: 1105-1115. 